The final publication is available at Springer via http://dx.doi.org/10.1007/ s10763-015-9659-9 
Running Head: MATH ANXIETY AND INTERACTIVITY

Interactivity Defuses the Impact of Mathematics Anxiety in Primary School Children

\author{
Michael Allen and Frédéric Vallée-Tourangeau* \\ Kingston University
}

\begin{abstract}
Author Note
Correspondence concerning this article should be addressed to Frédéric Vallée-Tourangeau, Department of Psychology, Kingston University, Kingston upon Thames. UNITED KINGDOM, KT1 2EE, f.vallee-tourangeau@kingston.ac.uk, tel: +44 (0)20 8417 2816, fax: +44 (0)20 8417 2388. We would like to thank teachers and students of Belgrove Senior Boys School, St. Vincent's Girls National School, Star of the Sea Boys National School, St. Mary’s Convent National School and St. Patrick’s Boys National School.
\end{abstract}




\begin{abstract}
Math anxiety impedes performance in simple arithmetic tasks. Anxiety constrains working memory capacity and particularly the attentional functions of the central executive. The experiment reported here explored how interactivity mitigated the impact of math anxiety on the performance of elementary school students with simple additions. It manipulated two independent variables. The first was the length of the additions - involving either 7 or 11 number tokens (the value of the tokens ranged from 1 to 20). The other was the level of interactivity: in the low interactivity condition participants could not touch or point to the number tokens that configured the sums, and in a high interactivity condition participants could manipulate the tokens as they saw fit in deriving their answer. The length of the addition had an impact on accuracy, with longer sums leading to poorer performance. However, overall, performance in the high interactivity condition was superior, in terms of accuracy, absolute calculation error and efficiency, than performance in the low interactivity condition. Mathematics anxiety significantly predicted performance in the low interactivity condition, but not in the high interactivity condition. These results suggest that working memory resources are augmented through interactivity with the physical problem presentation, defusing the impact of anxiety on performance.
\end{abstract}




\section{Interactivity Defuses the Impact of Math Anxiety in Primary School Children}

Anxiety undermining performance on mathematics tasks is termed mathematics anxiety (Richardson \& Suinn, 1972). Tobias and Weissbrod (1980, p. 65) describe it as "the panic, helplessness, paralysis and mental disorganisation that arises among some people when they are required to solve a mathematical problem." Anxiety related to mathematics can lead to incessant worry about failure in math performance (Ramirez, Gunderson, Levine, \& Beilock, 2013). This can often result in avoidance of numerical tasks (Krinzinger, Kaufmann, \& Willmes, 2009). Some studies have shown increased cortisol responses, related to stress, during performance on math tasks (Mattarella-Micke, Mateo, Kozak, Foster, \& Beilock, 2011). Lyons and Beilock (2010) found that even thoughts of doing math elicited a negative emotional response from math anxious students. Neuroimaging data show increased activity in regions associated with processing negative emotions (such as the amygdala) when performing arithmetic tasks in high math anxiety participants (Young, Wu, \& Menon, 2012)

Hembree (1990) conducted a meta-analysis of the effects of math anxiety. The studies reviewed demonstrate that high math anxiety individuals enrol in fewer math courses and earn lower grades when they do study math. Math anxiety was found to correlate — negatively — with learning, mastery and motivation, as well as with performance generally, grades in high school, enjoyment of math, and with motivation to study math in the future; highly anxious individuals took fewer math courses.

Expectations of success and the perceived value of math is reduced in students who report high anxiety levels (Eccles, Adler, Futterman, Goff, Kaczala, Meece, \& Midgley, 1983), and it can change goals related to achievement that students have within the domain of mathematics (Butler, 1999). Math anxious students tend to give up studying math as early as 
they can (Brown, Brown \& Bibby 2008). The anxiety is often carried into adulthood, limiting career opportunities (Cockroft, 1982; Smith, 2004). Miller and Bichsel (2004) found math anxiety to be a more important predictor of math performance when tested on basic and applied math than measures of working memory capacity. However, as avoidance tends to be one of the more pervasive long term effects (Ashcraft, 2002), it is possible that these effects and those documented by Hembree are a result of engaging less with mathematics.

In an attempt to determine whether reactions such as these specifically had an 'on-line' effect on an individual's math performance, that is, an effect on underlying cognitive processes as the individual completes a math related task, Ashcraft and Faust (1994) tested subjects over a range of simple and complex addition and multiplication problems. They found that anxious groups consistently performed worse in terms of errors made and latencies to solution, and this even with basic arithmetic operations. Wigfield and Meece (1988) in a study of elementary and secondary school students found that anxiety levels were similar in older and younger children, as well as in boys and girls. Ma and Xu (2004) found no sex differences in anxiety levels. However, the effect of this anxiety tends to be most noticeable in later childhood and adolescence, when the consequences of mathematics avoidance become more pronounced (Ramirez et al., 2013). At elementary level, before options which lead to mathematics avoidance are encountered, peers will have had similar exposures to mathematics. Thus, since underperformance among primary school children cannot yet be attributed to mathematics avoidance, this population offers an excellent opportunity to examine the links between anxiety, ability and performance. 


\section{Working Memory}

As a problem solving activity involving the rehearsal and storage of information (e.g., intermediate sums), the allocation of attentional resources, and the retrieval and selection of strategies, mental arithmetic implicates key working memory operations. Demands placed on working memory are proportional to the complexity of the task (LeFevre, DeStefano, Coleman, \& Shanahan, 2005). As a sum's operands grow larger, mental manipulation and storage of intermediate values become important. This is known as the 'problem-size' effect (Ashcraft \& Krause, 2007). Solutions of problems concerning small operands can be retrieved from memory (e.g., $3+5)$ whereas solutions with larger operands require a more complex strategy. In particular, executing an operation involving a 'carry over', places significant demands on working memory capacity (Ashcraft \& Kirk, 2001). Where more steps are involved, working memory processes are required to maintain intermediate values, to provide computational feedback and to manage attention across the required steps.

Researchers exploring the role of working memory on numerical cognition generally take as their starting point Baddeley's (1992) model of working memory, with its three interdependent sub-systems; a phonological loop, a visual spatial 'sketchpad', and a central executive. For example, Logie, Gilhooly and Wynn (1994) used memory loads that were designed to implicate specific working memory sub-systems in an attempt to investigate the subsequent effect of this load on problem solving. Using a dual-task methodology, they found that performance during problem solving showed a greater impairment by the concurrent memory load when the load was of the same modality as that in which the problem was presented (i.e., visual or verbal). The authors also found that random letter generation - which requires executive functions - disrupted mental arithmetic performance regardless of whether the 
problem was presented verbally or visually, and in a manner that consistently outweighed that of the memory loads. These results suggest a role for working memory, and in particular the central executive, in mathematical cognition, with operations differentially loading onto working memory components depending on problem type and presentation modality.

Seyler, Kirk and Ashcraft (2003) showed that participants with larger working memory capacity were less affected by dual task loads and showed smaller magnitude errors. Geary and Widaman (1992) found that skill in arithmetic problem solving was related to working memory capacity. Ashcraft and Kirk (2001) investigated the combined effect of math anxiety and working memory load on addition problems that involve a 'carry' operation. In combination with these problems participants performed a secondary letter memory task involving the maintenance of 2 or 6 letter strings in memory for the duration of the addition task. Error rates for highly math anxious individuals were only slightly more than for those with low math anxiety when asked to remember a 2-letter string. However, when this was increased to 6 letters, participants with higher levels of math anxiety produced significantly more errors than their low anxiety counterparts. Thus, as working memory demand increased, the performance of higher math anxious individuals suffered more.

Sarason (1988) suggested that anxiety has a disrupting effect on performance due to the interjecting of task-irrelevant thoughts. In turn, Eysenck and Calvo’s (1992) processing efficiency theory postulates that anxiety-related thoughts undermine the efficient allocation of processing resources, thus anxiety primarily affects the central executive. 'Attentional Control Theory’ (Eysenck, Derakshan, Santos, \& Calvo 2007) develops this idea further, describing the impact of anxiety specifically on the attentional control aspects of the central executive system, 
affecting both negative attentional control—inhibiting attention to task-irrelevant stimuli_-and positive attentional control—switching attention between and within tasks as appropriate.

\section{Interactivity}

A learning or problem-solving situation engineered in a manner that encourages a reasoner to interact with a physical problem presentation favours the development of an extended cognitive system (Wilson \& Clark, 2009); this system is defined in terms of the interweaving of the reasoner's internal resources and the resources offered by the environment. In this cognitive system, a dynamic problem representation emerges from the fluid interface of the reasoner and the environment as fashioned by the reasoner's actions. The control of behaviour and the allocation of attentional resources can also be distributed, at times reflecting endogenous factors, and at others more like a direct reaction to the environment's shifting configuration. As a result of the interactivity that couples a cognitive agent to his or her environment, working memory capacity and executive functions may be augmented.

Vallée-Tourangeau (2013) explored the role of interactivity in mental arithmetic. In that experiment, university undergraduates were asked to complete multi-number additions, and could either move number tokens that configured the problem or were required to remain stationary during the count. Interactivity enhanced accuracy, reducing calculation error, but it also improved problem solving efficiency. Thus, holding latency to solution constant, interactivity enhanced accuracy. In a related experiment, Carlson, Avraamides, Cary and Strasberg (2007) showed that if participants pointed at objects while counting them, the addition process was both faster and more accurate than if they did not point. 


\section{The Present Experiment}

If interactivity can augment working memory capacity, through the distributed storage of pertinent information and attentional control, participants may complete mental arithmetic problems more accurately and more efficiently. If math anxiety handicaps performance by highjacking working memory resources for the rehearsal and maintenance of anxiety-related thoughts, interactivity may defuse the impact of math anxiety on mental arithmetic performance by augmenting working memory capacity. The experiment reported here explored how the impact of math anxiety on performance in a simple mental arithmetic task could be defused through interaction with the problem presentation. Participants were asked to add up sets of numbered tokens either in a low interactivity context by placing their hands face down in front of them or in a high interactivity context in which they were encouraged to move the tokens — sets were composed of either 7 or 11 tokens. Addition in both set sizes is a relatively complex process as there are numerous steps involved, inevitably necessitating a 'carry' operation. The larger set size, however, should involve significantly more working memory resources in order to complete the count, resulting in poorer performance. Due to the potential cognitive off-load and working memory capacity release available through the manipulation of the physical presentation of the problem in the high interactivity condition, performance should be seen to improve in both set sizes, but particularly in the large set size condition as working memory capacity constraint would be more keenly felt during the addition of 11 numbers. Finally, as math anxiety has been shown to constrain working memory and impair performance, ratings of anxiety were expected to predict performance measures in the low interactivity but not in the high interactivity condition. 


\section{Method}

\section{Participants}

The study was conducted using 59 primary school students recruited from 5 different schools located in Dublin, Ireland, and Roscommon, Ireland. Students were in either $5^{\text {th }}$ or $6^{\text {th }}$ class, with ages ranging from $9-11$ years old, the average age being $10.1(S D=0.7)$. This age range was selected for study so that the effect of anxiety is more likely to be 'on-line' and less likely to be a consequence of math avoidance and therefore a deficiency in exposure or experience. Not all students within each class participated in the study due to time constraints in each school. Those students who did participate were selected according to the attendance list provided by the teacher, which was in alphabetical order. Nineteen participants were female, and 40 were male.

\section{Design}

The study used a 2 (set size) by 2 (interactivity) within subjects design with each participant attempting counting trials involving either 7 (short set size) or 11 (large set size) numbered tokens. In half the trials participants were asked to count the tokens without touching or moving them (low interactivity), and in the other half they were encouraged to move the tokens around as they pleased (high interactivity); the interactivity conditions order was counterbalanced across participants. Four counting trials were conducted per set size per condition, so that each participant were asked to complete 16 sums in total. Participants' response accuracy and latency for each addition were measured.

\section{Materials and Measures}

Mathematics anxiety. Each participant's mathematics related anxiety was recorded using a questionnaire adapted from the Math Anxiety Rating Scale- Elementary (Suinn, Taylor \& Edwards, 1988), which is a 26-item rating scale itself adapted from the original 98-item 
questionnaire (Suinn, 72). Questions were chosen so as to be most suitable for primary school children of the average age of participants used in this study; 9 questions were used so as not to burden the children. Participants used a 5-point scale ( $1=$ "not at all, 5 = "very much") to indicate how anxious the situation as described in each question would make them feel. Items addressed anxiety related to testing (e.g., "taking a math test in your class") and numerical anxiety (e.g., "deciding if this problem is right: $(3+4)+2=4+(2+3)$ " (see Humbree, 1990, for an analysis of the difference between these two anxiety subconstructs). Also included were items considered to represent both (e.g., "getting called on by a teacher to do a math problem on the board in front of the class").

Working memory capacity. Working memory was measured using a 'Total Digit Span' score, which is a composite of the forward and backward span tests from the 'Wechsler intelligence Scale for Children' - Third Edition (WISC-III; Wechsler, 1991) and as used in Ramirez et al. (2013). The forward digit span task is intended to measure immediate verbal short-term memory, where the backward digit span is more indicative of executive attention. A combined score of both forward and backward digit span was chosen as a working memory measure according to the logic outlined in Ramirez et al.: as WM is comprised of both memory and executive attention processes, the combined score aptly represents total working memory capacity. In the forward digit task, sequences of single-digit numbers, starting at 2 and increasing to a maximum 8 , were read out to the child at a rate of 1 digit per second. The child was asked to repeat the numbers back after each number had been read out. Two sequences of the same length were read out before moving to a longer sequence. The session ended either when the longest sequences were recalled or the participant failed to recall two sequences of the same length. The child's score was recorded as the number of digits correctly repeated back, with a maximum of 
16. The backward digit task was conducted in the same manner except that the child was asked to repeat the numbers in reverse order and the maximum sequence length was 7 numbers. The child's score was then recorded out of a maximum of 14 .

Arithmetic task. The counting trials were conducted using either 7 or 11 tokens picked at random from a deck of 20 tokens which were numbered from 1 to 20 . Pupils were required to add together the numbers represented by the series of tokens. Each token measured $3 \mathrm{~cm} \mathrm{x} 3 \mathrm{~cm}$. A stopwatch was used to record the time taken to provide an answer for each sum. Pupils completed 8 counting trials from the short set size first before progressing to 8 trials from the large set size. Within each set size, trials were conducted in blocks of 4 for each level of interactivity. Performance was measured in terms of the mean absolute calculation error from the actual total, the proportion of sums correct in each set size and interactivity condition and the mean efficiency. Efficiency was measured as the ratio of accuracy over time invested to complete the sum: accuracy was measured as the proportion of correct sums over total sums completed and time invested was determined by dividing the total time taken to complete the sums by the average of the time taken by the slowest $25 \%$ of participants. Therefore, for each set size within each interactivity condition the efficiency ratio was operationalized as:

(number of sums correct $\div$ total sums)

(time completing sums $\div$ average time by slowest quartile of participants completing same sums)

\section{Procedure}

Each session lasted between 20 and 30 minutes. The experiment started with a presentation of 7 numbered tokens chosen at random from a deck. Participants were asked to add together the numbers on the tokens and respond verbally with their answer when they were ready. In the low interactivity condition, participants were asked to count "without moving or touching the tokens in any way" and to keep their hands on the desk while counting. In the high interactivity 
condition, participants were encouraged to touch, move or group the tokens at will. Once four additions in one condition were completed, participants were given instructions pertaining to the second condition and 4 more sums of 7 tokens at a time were completed. The order in which the conditions were encountered was counterbalanced across all participants. This was then repeated in the same order, with 4 trials in each condition, but using 11 tokens at a time instead of 7 . Upon completion of all 16 trials, participants completed the working memory span tests.

\section{Results}

\section{Arithmetic Performance}

The average time taken to complete a sum from the short set group was 30.44 seconds ( $S D$ $=13.59)$ in the low interactivity condition and 33.48 seconds $(S D=12.90)$ in the high interactivity condition. Average time to complete sums from the larger set was 60.92 seconds $(S D=24.36)$ in the low interactivity condition and 59.59 seconds $(S D=23.19)$ when in the high interactivity condition. In a 2x2 repeated measures analysis of variance (ANOVA) the main effect of interactivity was not significant, $F<1$, however large set sizes took significantly longer to complete, $F(1,58)=233, p<.001$; The interaction between set sizes and interactivity condition was not significant, $F=1$.

Proportion Correct. The mean proportion of correct answers for the short additions was $.5(S D=.29)$ in the low interactivity condition and $.67(S D=.23)$ in the high interactivity condition (see the left panel of Fig. 1). Participants' accuracy declined with the longer sums: The mean proportion of correct answers was $.28(S D=.25)$ in the low interactivity condition and .52 $(S D=.30)$ in the low interactivity condition. A $2 \times 2$ repeated measures analysis ANOVA revealed a significant main effect of interactivity, $F(1,58)=35.4, p<.001$, a significant main 
effect of set size, $F(1,58)=36.5, p<.001$; the interaction, however, was not significant, $F(1,58)$

$=2.01, p=.162$.

Absolute Calculation Error. In the low interactivity condition, the mean absolute calculation error for the shorter additions was $4.06(S D=3.3)$, but declined to $2.36(S D=2.5)$ in the high interactivity condition (see the middle panel of Fig. 1). In the larger set size of 11 tokens the average absolute error recorded in the low interactivity condition was $9.98(S D=8.37)$ and $5.19(S D=5.9)$ in the high interactivity condition. A $2 \times 2$ repeated measures ANOVA revealed a main effect of interactivity $F(1,58)=26.2, p<.001$, a main effect of set size, $F(1,58)=38.5, p<$ .001 , as well as a significant interaction, $F(1,58)=6.4, p=.014$.
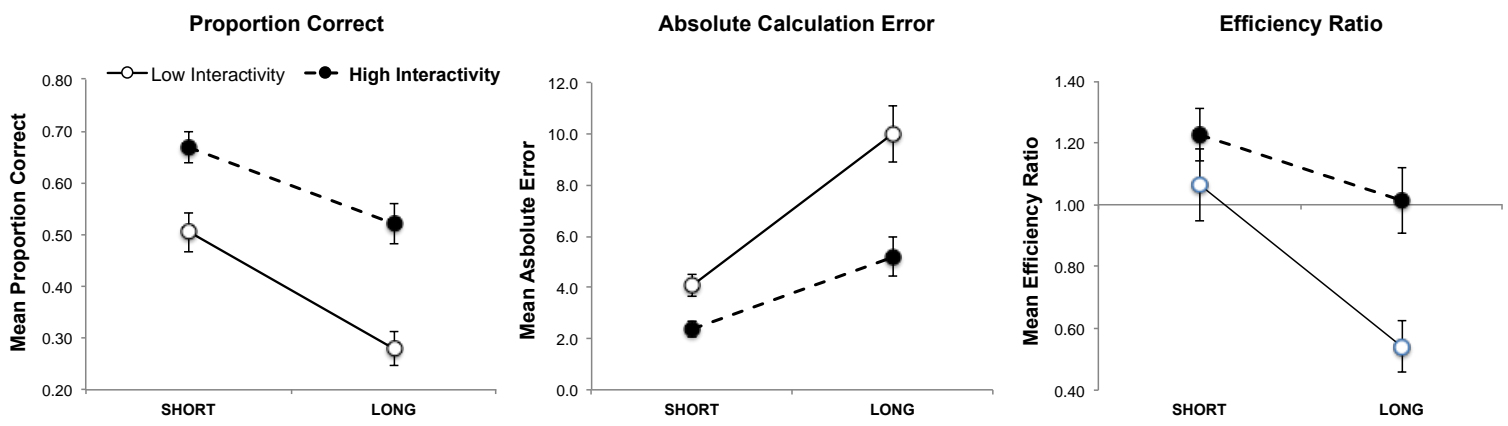

Figure 1. Mean proportion correct answers (left panel), mean absolute calculation error (middle panel), and mean efficienty ratios (right panel) for the short (7 numbers) and long (11 numbers) additions in the low interactivity condition (white bars) and in the the high interactivity condition (dark bars). Error bars are standard errors of the mean.

Efficiency. Mean efficiency ratios with the shorter sums were $1.06(S D=.89)$ and 1.23 $(S D=.65)$ in the low and high interactivity conditions, respectively (see the right panel of Fig. 1). These ratios were $.54(S D=.64)$ in the low interactivity condition and $1.01(S D=.82)$ in the high interactivity condition for the longer sums. Thus in the low interactivity condition, efficiency dropped substantially with longer sums with participants investing a lot of time to derive answers that were often incorrect. In contrast, efficiency remained above 1 in the high interactivity condition even with longer sums: Thus interactivity enabled participants to get a fair 
return on accuracy as a function of the time invested in computing the sums. A $2 \times 2$ repeated measures ANOVA confirmed these observations: The main effect of interactivity, $F(1,58)=$ $13.96, p<.001$, and the main effect of set size, $F(1,58)=16.901, p<.001$, were significant, as was the interaction between set size and interactivity, $F(1,58)=6.591, p=.013$.

\section{Predictors of Performance}

The mean math anxiety rating score across all participants was $15.15(S D=4.79)$. The mean recall score in the Total Digit Span was $16.9(S D=3.13)$. Math anxiety correlated negatively with working memory span, $r=-.363, p=.005$ (see Table $1 ; d f=57$ unless noted otherwise).

Table 1: Correlation matrix involving mathematics anxiety, working memory capacity and mental arithmetic performance averaged across both set sizes within each interactivity condition $(d f=57)$.

$\begin{array}{rrrrrrr} & 1 & 2 & 3 & 4 & 5 & 6 \\ & \text { M-ANX } & \text { SPAN } & \text { ERR-L } & \text { ERR-H } & \text { EFF-L } & \text { EFF-H } \\ 1 & - & -.363^{* *} & .409^{* *} & .120 & -.380^{* *} & -.185 \\ 2 & & - & -.463^{* *} & -.355^{* *} & .287^{*} & .239 \\ 3 & & & - & .338^{* *} & -.554^{* *} & -.328^{*} \\ 4 & & & & - & -.334^{* *} & -.593^{* *} \\ 5 & & & & & - & .476^{* *} \\ 6 & & & & & & -\end{array}$

$* p<.05 ; * * p<.01 . \mathbf{M}-\mathbf{A N X}=$ Mathematics anxiety scores; $\mathbf{S P A N}=$ Computation span scores; ERR-L $=$ Average absolute calculation error in the low interactivity condition; $\mathbf{E E R}-\mathbf{H}=$ Average absolute calculation error in the high interactivity condition; EFF-L = Average efficiency in the low interactivity condition; EFF-H = Average efficiency ratio in the high interactivity condition.

Absolute error. The average absolute error recorded across both set sizes in the low interactivity condition correlated positively with math anxiety, $r=.409, p=.001$. A similarly 
strong but negative correlation was found between these scores and working memory span, $r=$ $-.463, p<.001$. In the high interactivity condition, however, there was no significant correlation between anxiety levels and average absolute deviations, $r=.12, p=.363$. The significant correlation with working memory span remained, however, $r=-.355, p=.006$.

Efficiency. Efficiency ratios, averaged across the short and large set sizes, correlated significantly with math anxiety, $r=-.38, p=.003$ and working memory span $r=.287, p=.028$ in the low interactivity condition. However, in the high interactivity condition efficiency was not significantly correlated with either anxiety, $r=-.185, p=.161$, or working memory span, $r=$ $.239, p=.069$.

\section{Discussion}

The present experiment illustrated the role of interactivity in enhancing mental arithmetic performance, and more important how interactivity can defuse the impact of math anxiety on performance. In a comprehensive study on problem complexity and working memory, LeFevre, DeStefano, Coleman and Shanahan (2005) found a direct relation between working memory demands and the complexity of arithmetic problems. Demands on resources increase as the operands in a math problem increase in size, the number of steps needed to reach a solution increase, or when a 'carry' operation is necessary for solution. The significant effect of set size reported here is indicative of the higher levels of complexity involved in counting 11 numbers rather than 7 . There were more carry over operations, larger numbers involved and more steps to reach the total with sums of 11 numbers. These factors increase both the working memory resources required and the potential for making errors.

Anxiety affects performance by constraining the resources available and impairing ‘processing efficiency' (Eysenck \& Calvo, 1992). Therefore with limited working memory 
resources, participants who experience anxiety will also experience impaired performance as a result, particularly on arithmetic problems of greater complexity. Interacting with a fluid problem presentation in the high interactivity condition changes the terrain of cognition considerably: For one complementary and epistemic actions lighten the working memory load, but interactivity may also have a transformative impact on cognition. That is, shaping and reshaping a problem presentation, by grouping some tokens and not others, may cue different arithmetic strategies, strategies that would have been more difficult to identify or retrieve from long term memory in the low interactivity condition. Consistent with previous findings with adult participants (ValléeTourangeau, 2013; Vallée-Tourangeau, Sirota \& Villejoubert, 2013), the experiment reported here demonstrated that a high level of interactivity fostered greater accuracy and efficiency. The significant interaction between problem difficulty — or set size — and interactivity level confirmed that when the problems were easier, interactivity had a marginal impact on performance. This is because participants' internal resources could manage the problem difficulty with or without interactivity. However, with the longer additions, the absence of interactivity significantly depressed performance; in contrast, performance in the high interactivity condition was considerably more resilient.

Previous studies have reported similar performance-enhancing effects wrought by interactivity. Carlson, Avraamides, Cary and Strasberg (2007) found that pointing while counting arrays of asterisks increased both speed and accuracy of count. In this case, pointing is an example of a 'complementary strategy' (Kirsh, 1995); it augments cognition by acting as a place marker for the count, thereby removing the requirement for working memory to remember its place, and as a tool for managing the allocation of attentional resources. Kirsh (2013b) suggests that pointing works to anchor mental processes onto external features, increasing the 
effectiveness of the mental strategy employed during counting by relieving the burden of attentional management from internal working memory. Touching the numbered tokens as they are counted provides declarative representations of the numbers, allowing for more robust storage in short term memory. Equally, the hands can support procedural aspects of the task such as coordination and the binding of declarative information to its functional role (Carlson et al., 2007). In this experiment, participants were often seen to touch each number as they were being counted and then move them to one side out of their immediate view. Touching the number at each count point cues declarative information, draws attention to that number, and the subsequent removal of the number acts as a procedural aid; the participant no longer needs to remember not to count that number. Complementary actions are also observed when participants engage in mental abacus. In Frank and Barner (2012) elementary school pupils were taught to use an abacus for simple arithmetic, and were trained until they were relatively proficient. In a second phase, they were presented new problems, without the abacus. In completing the problems, they used their fingers and performed hand movements as if they were interacting with beads. Commenting on Frank and Barner’s findings, Kirsh (2013b, p. 21) writes: “(...) gesturing is not simply an epiphenomenon, that is, an unnecessary accompaniment to the mental operations that do the real work. Hand motions seem to improve mental simulation."

Another common strategy witnessed during the experiment was the grouping of numbers together to provide clusters of numbers whose count is easily remembered. This effectively reduced the quantity of numbers to be counted. Where for example, 7 and 13 were grouped together, this group can be easily seen to represent ' 20 ', and counted as one number. This kind of grouping is an 'epistemic' action that improves the likelihood of deriving the correct sum by creating a more cognitively congenial task layout. These actions "change the input into an 
agent's information-processing system" (Kirsh \& Maglio, 1994, p. 29), requiring less computational power, less working memory capacity, and thereby enhancing efficiency.

\section{Math Anxiety and Interactivity}

In the low interactivity condition, pupils with higher levels of math anxiety made larger counting errors. However, when allowed to interact with the tokens, this correlation disappeared: math anxiety was no longer a predictor of error. Similarly, in the low interactivity condition, anxiety ratings correlated negatively with efficiency - pupils with higher levels of math anxiety demonstrated less efficiency over both set sizes than their lower anxiety counterparts. As with calculation error, this correlational pattern was not observed in the high interactivity condition: Calculation efficiency was unrelated to math anxiety level. These findings suggest that in the high interactivity condition, math anxiety no longer had an effect on a participant's ability to count effectively.

That performance measures were not at ceiling level in the interactive condition among low anxious participants indicates that the non-significant correlations with anxiety was not simply because the task was easier. Ratings of working memory capacity have been shown to predict performance on cognitive tasks such as mental arithmetic (Engle, 2002). In the low interactivity condition, working memory capacity correlated negatively with the average absolute calculation errors. In the high interactivity condition, despite there no longer being a correlation with math anxiety, working memory remained a significant predictor of performance. This suggests that the problems still retained a degree of difficulty and that ability, as measured by working memory capacity, remained a contributor to performance. The lack of a correlation with math anxiety suggests that performance was depressed in the low interactivity condition for participants with higher levels of math anxiety, and that this depression was relieved in the high 
interactivity condition allowing performance to better reflect true ability. Efficiency too was marginally correlated with working memory in the high interactivity condition. Tests in which participants experience pressure, or anxiety, are thought to produce scores which tend to underestimate their levels of math ability. In this case, interactivity reduced or even negated completely this underestimation: anxious pupils gained more from the interactions relative to performance in the low interactivity condition than their non-anxious counterparts.

Interacting with the external environment affords access to greater cognitive resources, mitigating the effect of anxiety. Less clear, however, is whether anxiety itself is mitigated in the high interactivity condition. Since the experiment employed a repeated measures design, participants provided an anxiety score that was correlated with performance in both interactivity conditions. However, the exact level of anxiety arousal in each condition is unknown. In the high interactivity condition, the cognitive and strategic gains from interacting with a modifiable problem presentation may have simply released extra working memory resources which can overcome capacity depletion caused by rehearsing anxiety-related thoughts. However, it seems unlikely that performance could reach normal levels in the interactivity condition while participants still experienced the same levels of anxiety arousal. For one thing, achievement in math has been shown to accompany a reduction in anxiety levels (Hembree, 1990) - as participants find reassurance through the more straightforward solution process which accompanies a cognitively congenial manipulation of the presentation, it is likely that they will gain in confidence and begin to relax.

A further clue can be found from a closer inspection of the impact of anxiety on the central executive's attentional systems. Attentional control is thought to consist of both 'topdown' goal-driven processes, and 'bottom-up' stimulus-driven processes (Yantis, 1998). The 
former is influenced by the individual's current goals and knowledge, it pro-actively attempts to achieve an end by imposing a strategy on the central executive processing. The latter is reactive and influenced by sensory events which are behaviourally relevant, particularly when they are salient and unattended (Corbetta \& Shulman, 2002). These two systems of attentional control run concurrently and interact with each other. Attentional control theory proposes that anxiety enhances the bottom-up system, causing an excessive level of attention to be given to sensory stimuli which may not be task relevant. In a system of limited capacity, this causes a decrease in attention given to the task itself. It has been proposed (Miyake et al., 2000; Friedman \& Miyake, 2004) that the central executive attentional systems involve an inhibition function, which disrupts attention to irrelevant stimuli, a shifting function, which moves attention as required, and an updating function which involves monitoring and updating working memory representations. Empirical evidence has been provided by Derakshan and Eysenck (2009) which suggests that anxiety primarily effects the inhibition and shifting functions.

If anxiety affects performance by harnessing attentional function away from the task itself, then it is possible that these effects may be felt due to constraints in attentional capacity and not in working memory as a whole. The increased resources available due to off-load onto the physical environment will not necessarily reverse a performance impairment if anxiety continues to causes an impairment of the central executive's ability to inhibit unnecessary information and shift attention suitably with the task at hand.

Interactivity directs attention. The fluid problem presentation continually provides fresh cues. Manipulating the tokens draws attention to them, moving the tokens strategically helps to manage awareness of the current state of the problem and the path towards a solution. Relative to the low interactivity condition the perceptive input from the problem presentation is more salient 
and resilient. When cognition is affected by anxiety-related thoughts and memories, the individual experiences an increase in bottom-up attention, reflecting a growing desire to escape or avoid the task as it is the source of anxiety. In the low interactivity condition, a highly anxious individual will have conflicting demands within the attentional system which will serve to disrupt the task solution trajectory, causing the solver to lose his or her place, forget to count a token, or a number of other potential errors. When interacting with the problem presentation however, the persistently salient offerings of the physical representation serve to anchor attention, and thus counteract the attentional demands from the anxiety-related material. This allows the solver to maintain focus and proceed to solution more efficiently. Thus, while much work remains to be done to identify precisely how interactivity enhances and transforms mental arithmetic performance, an important implication of our findings for primary school education is to train math anxious pupils solve problems in a more interactive context. Doing so will likely defuse or eliminate the deleterious impact of anxiety and enhance the children's performance. There are felicitous consequences, of course, to experiencing success at a task: it raises confidence and self-efficacy and may reduce or eliminate avoidance as a coping strategy, encouraging pupils to work harder and longer on mathematics. 


\section{References}

Ashcraft, M. H. (2002). Math anxiety: Personal, educational, and cognitive consequences. Current Directions in Psychological Science, 11, 181-185.

Ashcraft, M. H., \& Faust, M. W. (1994). Mathematics anxiety and mental arithmetic performance: An exploratory investigation. Cognition \& Emotion, 8, 97-125.

Ashcraft, M. H., \& Kirk, E. P. (2001). The relationships among working memory, math anxiety, and performance. Journal of experimental psychology: General, 130, 224 - 237.

Ashcraft, M. H., \& Krause, J. A. (2007). Working memory, math performance, and math anxiety. Psychonomic Bulletin \& Review, 14, 243-248.

Baddeley, A. (1992). Working memory. Science, 255(5044), 556-559.

Brown, M., Brown, P., \& Bibby, T. (2008). "I would rather die": Reasons given by 16-year-olds for not continuing their study of mathematics. Research in Mathematics Education, 10, 318.

Butler, R. (1999). Information seeking and achievement motivation in middle childhood and adolescence: The role of conceptions of ability. Developmental Psychology, 35, 146-163.

Carlson, R. A., Avraamides, M. N., Cary, M., \& Strasberg, S. (2007). What do the hands externalize in simple arithmetic? Journal of Experimental Psychology: Learning, Memory, and Cognition, 33, $747-756$.

Corbetta, M., \& Shulman, G. L. (2002). Control of goal-directed and stimulus-driven attention in the brain. Nature reviews neuroscience, 3, 201-215.

Derakshan, N., \& Eysenck, M. W. (2009). Anxiety, processing efficiency, and cognitive performance. European Psychologist, 14, 168-176. 
Eccles, J., Adler, T. F., Futterman, R., Goff, S. B., Kaczala, C. M., Meece, J., and Midgley, C. (1983). Expectancies, values and academic behaviors. In J. T. Spence (Ed.), Achievement and achievement motives (pp. 95-121). San Francisco: W. H. Freeman.

Engle, R. W. (2002). Working memory capacity as executive attention. Current Directions in Psychological Science, 11, 19-23.

Eysenck, M. W., \& Calvo, M. G. (1992). Anxiety and performance: The processing efficiency theory. Cognition \& Emotion, 6, 409-434.

Eysenck, M. W., Derakshan, N., Santos, R., \& Calvo, M. G. (2007). Anxiety and cognitive performance: Attentional control theory. Emotion, 7, 336-353.

Faust, M. W. (1996). Mathematics anxiety effects in simple and complex addition. Mathematical Cognition, 2, 25-62.

Frank, M, \& Barner, D. (2012). Representing exact number visually using mental abacus. Journal of Experimental Psychology: General, 141, 134-149.

Friedman, N. P., \& Miyake, A. (2004). The relations among inhibition and interference control functions: a latent-variable analysis. Journal of Experimental Psychology: General, 133, 101-135.

Geary, D. C., \& Widaman, K. F. (1992). Numerical cognition: On the convergence of componential and psychometric models. Intelligence, 16, 47-80.

Hembree, R. (1990). The nature, effects, and relief of mathematics anxiety. Journal for Research in Mathematics Education, 21, 33-46.

Kirsh, D. (1995). Complementary strategies: Why we use our hands when we think. In Proceedings of the Seventeenth Annual Conference of the Cognitive Science Society (pp. 212-217). Mahwah, NJ: Lawrence Erlbaum Associates. 
Kirsh, D. (2013a). Embodied cognition and the magical future of interaction design. $A C M$ Transactions on Computer-Human Interactions, 20(1), 1-30.

Kirsh, D. (2013b). Thinking with external representations. In S. J. Cowley, \& F. ValléeTourangeau (Eds.), Cognition beyond the brain: Interactivity, cognition and human artifice (pp. 171-194). Dordrecht: Springer.

Kirsh, D., \& Maglio, P. (1994). On distinguishing epistemic from pragmatic action. Cognitive science, $18,513-549$.

Krinzinger, H., Kaufmann, L., \& Willmes, K. (2009). Math anxiety and math ability in early primary school years. Journal of Psychoeducational Assessment, 27, 206-225.

LeFevre, J., DeStefano, D., Coleman, B., \& Shanahan, T. (2005). Mathematical cognition and working memory. Handbook of Mathematical Cognition, 361-378.

Logie, R. H., Gilhooly, K. J., \& Wynn, V. (1994). Counting on working memory in arithmetic problem solving. Memory \& Cognition, 22, 395-410.

Lyons, I. M., \& Beilock, S. L. (2012). Mathematics anxiety: Separating the math from the anxiety. Cerebral Cortex, 22, 2102-2110.

Ma, X., \& Xu, J. (2004). The causal ordering of mathematics anxiety and mathematics achievement: a longitudinal panel analysis. Journal of Adolescence, 27, 165-179.

Mattarella-Micke, A., Mateo, J., Kozak, M. N., Foster, K., \& Beilock, S. L. (2011). Choke or thrive? The relation between salivary cortisol and math performance depends on individual differences in working memory and math-anxiety. Emotion, 11, 1000-1005.

Miller, H., \& Bichsel, J. (2004). Anxiety, working memory, gender, and math performance. Personality and Individual Differences, 37, 591-606. 
Miyake, A., Friedman, N. P., Emerson, M. J., Witzki, A. H., Howerter, A., \& Wager, T. D. (2000). The unity and diversity of executive functions and their contributions to complex "frontal lobe" tasks: A latent variable analysis. Cognitive psychology, 41, 49-100.

Ramirez, G., Gunderson, E. A., Levine, S. C., \& Beilock, S. L. (2013). Math anxiety, working memory, and math achievement in early elementary school. Journal of Cognition and Development, 14, 187-202.

Richardson, F. C., \& Suinn, R. M. (1972). The Mathematics Anxiety Rating Scale: Psychometric data. Journal of Counseling Psychology, 19, 551-554.

Sarason, I. G. (1988). Anxiety, self-preoccupation and attention. Anxiety Research, 1, 3-7.

Seyler, D. J., Kirk, E. P., \& Ashcraft, M. H. (2003). Elementary Subtraction. Journal of Experimental Psychology: Learning, Memory, and Cognition, 29, 1339-1352.

Smith, A. (2004). Making mathematics count: the report of Professor Adrian Smith's inquiry into post-14 mathematics education. http://dera.ioe.ac.uk/4873/1/MathInquiryFinalReport.pdf

Suinn, R. M., Taylor, S., \& Edwards, R. W. (1988). Suinn mathematics anxiety rating scale for elementary school students (MARS-E): Psychometric and normative data. Educational and Psychological Measurement, 48, 979-986.

Tobias, S., \& Weissbrod, C. (1980). Anxiety and mathematics: An update. Harvard Educational Review, 50, 63-70.

Vallée-Tourangeau, F. (2013). Interactivity, efficiency, and individual differences in mental arithmetic. Experimental Psychology, 60, 302-311.

Vallée-Tourangeau, F., Sirota, M., \& Villejoubert, G. (2013). Reducing the impact of math anxiety on mental arithmetic: The importance of distributed cognition. In M. Knauff, M. Pauen, N. Sebanz, \& I. Wachsmuth (Eds.), Proceedings of the Thirty-Fifth Annual 
Conference of the Cognitive Science Society (pp. 3615-3620). Austin, TX: Cognitive Science Society.

Wechsler, D. (1991). WISC-III: Wechsler intelligence scale for children. San Antonio, TX: Psychological Corporation.

Wigfield, A., \& Meece, J. L. (1988). Math anxiety in elementary and secondary school students. Journal of Educational Psychology, 80, 210-216.

Wilson, R. A., \& Clark, A. (2009). How to situate cognition: Letting nature take its course. In P. Robbins \& M. Aydede (Eds.), The Cambridge handbook of situated cognition (pp. 5577). Cambridge, UK: Cambridge University Press.

Yantis, S. (1998). Control of visual attention. Attention, 1, 223-256.

Young, C. B., Wu, S. S., \& Menon, V. (2012). The neurodevelopmental basis of math anxiety. Psychological Science, 23, 492-501. 